\title{
Introduction to the DISRUPT postprandial database: subjects, studies and methodologies
}

\author{
Kim G. Jackson • Dave T. Clarke • Peter Murray • \\ Julie A. Lovegrove $\cdot$ Brendan O'Malley • \\ Anne M. Minihane $\cdot$ Christine M. Williams
}

Received: 12 May 2009/Accepted: 14 September 2009/Published online: 29 September 2009

(C) Springer-Verlag 2009

\begin{abstract}
Dysregulation of lipid and glucose metabolism in the postprandial state are recognised as important risk factors for the development of cardiovascular disease and type 2 diabetes. Our objective was to create a comprehensive, standardised database of postprandial studies to provide insights into the physiological factors that influence postprandial lipid and glucose responses. Data were collated from subjects $(n=467)$ taking part in single and sequential meal postprandial studies conducted by researchers at the University of Reading, to form the DISRUPT (DIetary Studies: Reading Unilever Postprandial Trials) database. Subject attributes including age, gender, genotype, menopausal status, body mass index, blood pressure and a fasting biochemical profile, together with postprandial measurements of triacylglycerol (TAG), non-esterified fatty acids, glucose, insulin and TAG-rich lipoprotein composition are recorded. A particular strength of the studies is the frequency of blood sampling, with on average 10-13 blood samples taken during each postprandial assessment, and the fact that identical test meal protocols were used in a number of studies, allowing pooling of data to increase statistical power. The DISRUPT database is the most comprehensive postprandial
\end{abstract}

K. G. Jackson ( $₫)$ · J. A. Lovegrove · A. M. Minihane ·

C. M. Williams

Hugh Sinclair Unit of Human Nutrition, Department of Food and Nutritional Sciences, University of Reading, PO Box 226, Whiteknights, Reading RG6 6AP, UK

e-mail: k.g.jackson@ reading.ac.uk

D. T. Clarke

Visualize Systems Ltd, Buckingham MK18 1WZ, UK

P. Murray · B. O’Malley

Unilever Discover, Colworth Science Park,

Sharnbrook MK44 1LQ, UK metabolism database that exists worldwide and preliminary analysis of the pooled sequential meal postprandial dataset has revealed both confirmatory and novel observations with respect to the impact of gender and age on the postprandial TAG response. Further analysis of the dataset using conventional statistical techniques along with integrated mathematical models and clustering analysis will provide a unique opportunity to greatly expand current knowledge of the aetiology of inter-individual variability in postprandial lipid and glucose responses.

Keywords Cardiovascular disease - Genotype · Mathematical modelling - Single and multiple test meals . Postprandial lipid metabolism · Glucose

\section{Introduction}

Recent evidence has shown elevated postprandial triacylglycerol (TAG) to be an independent risk factor for cardiovascular disease (CVD) [2, 29], and more discriminatory than fasting TAG. Increased levels of TAG-rich lipoproteins (TRLs; chylomicrons and very low density lipoprotein) in both the fed (postprandial) and fasted states is the metabolic driver of a combined dyslipidaemia referred to as the atherogenic lipoprotein phenotype, and a clinical feature of type 2 diabetes and the metabolic syndrome. The atherogenic impact of TAG is thought to be mediated in part by a reduction in high density lipoprotein cholesterol (HDL-C) concentration and a decrease in the size of low density lipoprotein (LDL) towards the smaller more atherogenic LDL-III and LDL-IV particles [23]. Importantly, the dyslipidaemia is highly correlated with excess body weight and because of the increasing 
prevalence of both overweight and obesity, its occurrence in the UK, especially in middle-aged men and women, is extremely common [4]. Despite the widespread manifestation of this lipid abnormality in adult populations of most developed countries, its aetiology and capacity for modification by lifestyle factors such as diet remains unclear.

As a result of the frequency of meal and snack consumption throughout the day, individuals are exposed to elevated circulating TAG levels for on average $18 \mathrm{~h}$ per day. The magnitude and duration of the postprandial lipaemic response is influenced by a number of metabolic processes, including the rate of secretion of TRL from the intestine and the liver, the activity of enzymes involved in the processing of TRLs and the rate of clearance of TRL remnants by receptor mediated processes [25]. Since Zilversmit [42] hypothesised that TRL remnants were atherogenic in 1979, there has been considerable research interest into the acute and chronic impact of dietary fat composition on postprandial lipaemia. However, determination of the postprandial response is complex and the lack of standardisation of methodologies, in particular test meal size and composition, between different studies and research groups has hampered the elucidation of lifestyle and physiological factors which influence postprandial lipaemia [23]. In addition, the degree of certainty that can be placed on summary measures of the lipaemic response is often limited by small subject numbers and infrequent blood sampling during the postprandial period.

The DISRUPT (DIetary Studies: Reading Unilever Postprandial Trials) project has collated data from subjects taking part in single meal $(n=162)$ and sequential (two) meal $(n=305)$ postprandial studies conducted over the past 16 years by investigators at the University of Reading. This detailed postprandial dataset has been compiled into a single standardised database, which is the largest such in existence to date. This extensive dataset will enable (a) comprehensive analyses of the impact of age, gender, body mass index, genotype and the fasting metabolite profile on the inter-individual variability of the postprandial lipaemic response and (b) facilitate the development of predictive and integrative mathematical models of postprandial metabolic responses to mixed meals.

This paper will introduce the study protocols, subjects, clinical and biochemical measures, and postprandial time course data contained within the postprandial database and detail opportunities for enhancing the scientific value of such pooled datasets through statistical analyses and mathematical modelling methodologies. In addition, preliminary findings from the statistical analysis of the pooled sequential meal postprandial dataset will also be presented.

\section{Postprandial database}

The creation of the DISRUPT database is a joint initiative between researchers in the Hugh Sinclair Unit of Human Nutrition at the University of Reading and the Systems Biology of Lipid Metabolism group at Unilever, the purpose of which is to maximise the scientific output and value from already completed postprandial studies at Reading and to identify gaps in our current knowledge.

The database has facilities for data entry, analysis, and exporting through a user friendly web interface and electronic data capture capabilities to enable the recording of future, as well as past, studies in a consistent, comprehensive and standardised manner. All key information are entered using a double data entry approach to minimise errors, with any discrepancies being clearly highlighted on the screen before the study status can be finalised on the database. All entries use consistent units and standardised test parameter/subject attribute names, which are stored in a relational database $[5,6]$. Analysis and charting tools are contained within the database, which include the facility to compare and filter groups of data across one or a number of postprandial studies at a time. Reporting options are available for the subject attribute information, genotype and postprandial metabolite concentrations. Security levels restrict access to the data via user roles and all data are anonymous.

The electronic capture functionality was designed to address the common challenges faced by the two research groups, in both comparing the results of dietary interventions in different studies and utilising such studies for further analysis and modelling. There is also scope to upgrade this database of studies conducting by researchers at Reading to include postprandial data collected by other groups working in this field.

\section{Study protocols and methodology}

According to specific inclusion criteria, participants for both the single and sequential meal postprandial studies were recruited from the local population (Table 1). Initial screening of the subjects was performed by phone using a health and lifestyle questionnaire. Information was collected on personal medical history and family history of CVD in first-degree relatives and siblings, smoking and alcohol habits and physical activity levels. All prescribed medications were recorded including intake of dietary supplements. For female participants, information on menopausal status was self-reported, with postmenopausal categorised by amenorrhea for at least 12 months.

If suitable, based on the health and lifestyle questionnaire performed over the phone, subjects were then invited 
Table 1 General inclusion and exclusion criteria

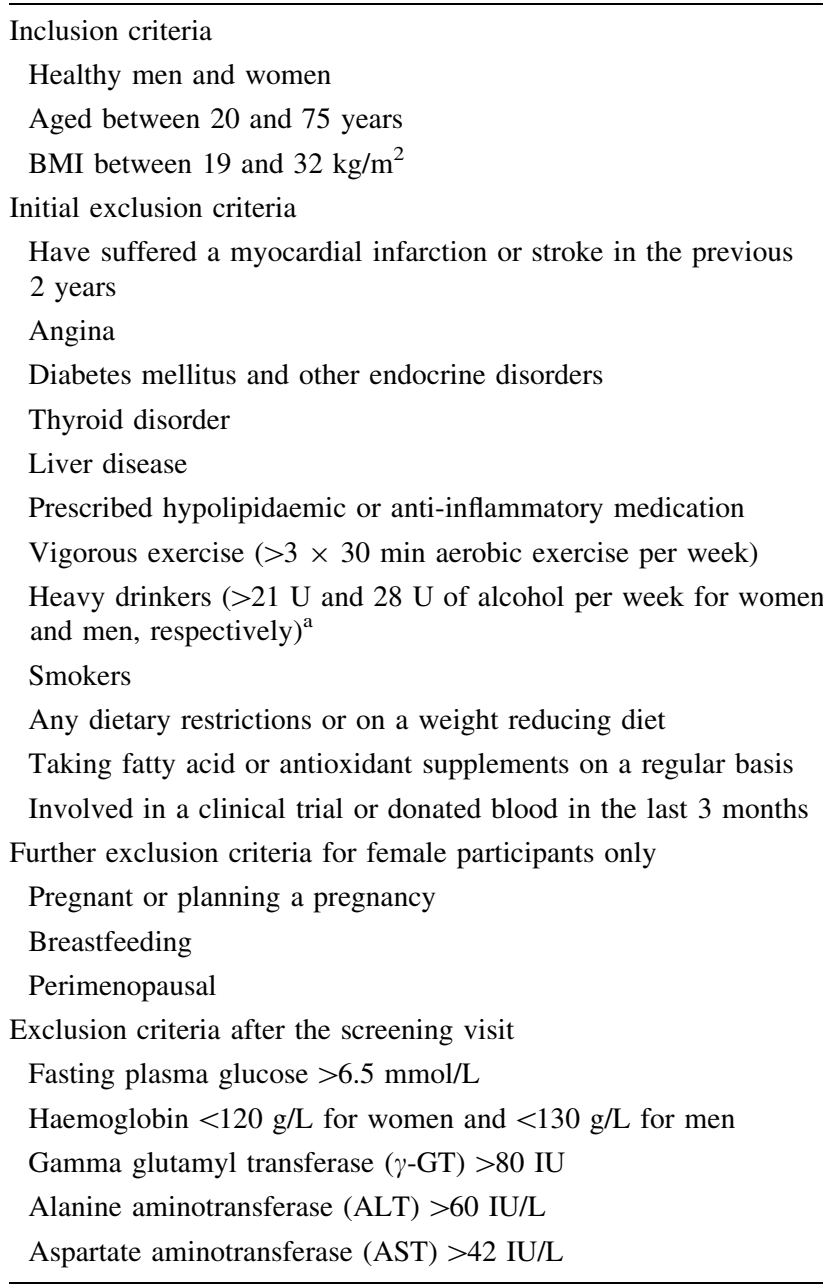

${ }^{a}$ One unit is equivalent to eight grams of alcohol

to the investigation unit for the measurement of height, weight, blood pressure and to provide a fasting blood sample for the analysis of lipids, glucose, liver function tests and haemoglobin. LDL cholesterol (LDL-C) was calculated using the Friedewald formula [11]. Blood pressure was measured using an OMRON MX2 automatic blood pressure monitor and body mass index (BMI) was calculated from the height and weight measurements.

\section{Sequential test meal protocols}

Postprandial protocols employing two test meals (sequential meal design) have been used in eight of the fifteen studies conducted by investigators at the University of Reading. This type of postprandial protocol has been used to determine (a) the effect of chronic dietary fat manipulation or cereal/non-digestible carbohydrate supplement on the postprandial lipaemic response (studies 1-6, $n=257$ subjects) [3, 10, 24, 26, 28], (b) influence of body size and shape on the postprandial lipaemic response (study 7 , $n=28$ subjects) [27, 39] and (c) the acute impact of specific meal fatty acids in the first meal on the postprandial response to the second meal (studies 8 and $9, n=20$ subjects) [16-18, 32-34]. An overview of the study designs are shown in Fig. 1. A key feature of studies 1-6 which we will now refer to as the "sequential meal postprandial dataset" is the use of the same test meals and frequency of blood sampling during all of the postprandial investigations. This similarity in protocol design has allowed the data to be pooled together easily, which increases the statistical power of this dataset. This study protocol is described in detail below ("Sequential meal postprandial dataset"), with preliminary findings from this dataset presented in "Preliminary findings from the sequential meal postprandial dataset" section.

For the study examining the effect of body shape and size on postprandial lipaemia (study 7) and the two studies examining the impact of meal fatty acid composition on the postprandial response to the second meal (studies 8 and 9), the test meal composition and protocols were unique to these individual studies and are summarised in Fig. 1. More detailed information of the subject groups, study protocols and postprandial measurements can be obtained from the study publications [16-18, 27, 32-34, 39].

\section{Sequential meal postprandial dataset}

In order to analyse the influence of subject attributes such as age, gender, menopausal status, genotype, BMI and fasting lipids on the inter-individual variability of the postprandial lipaemic response, the baseline data from six individual studies employing the same sequential test meal protocol were pooled together for this postprandial dataset (Fig. 1). All subjects were recruited using similar inclusion/exclusion criteria (Table 1) and at the time of the baseline postprandial investigation, all individuals were following their habitual diet and had not commenced the relevant dietary intervention. Healthy adults living in the UK aged 22-72 years with a BMI between 19 and $32 \mathrm{~kg} / \mathrm{m}^{2}$ and fasting glucose $<6.5 \mathrm{mmol} / \mathrm{L}$ were recruited. The studies were approved by the University of Reading Ethics and Research Committee and the West Berkshire Health Authority Ethics Committee, and written informed consent was obtained from the subjects before the study began.

Postprandial protocol On the day prior to the postprandial investigation, subjects were asked to abstain from alcohol and organised exercise regimens. A low fat evening meal ( $<10 \mathrm{~g}$ fat) was provided to standardise short-term fat intake. After a 12-h overnight fast, the subjects attended the investigation unit where an indwelling cannula was inserted into the anticubital vein of the forearm under local anaesthetic (1\% lignocaine) and two fasting blood samples were 


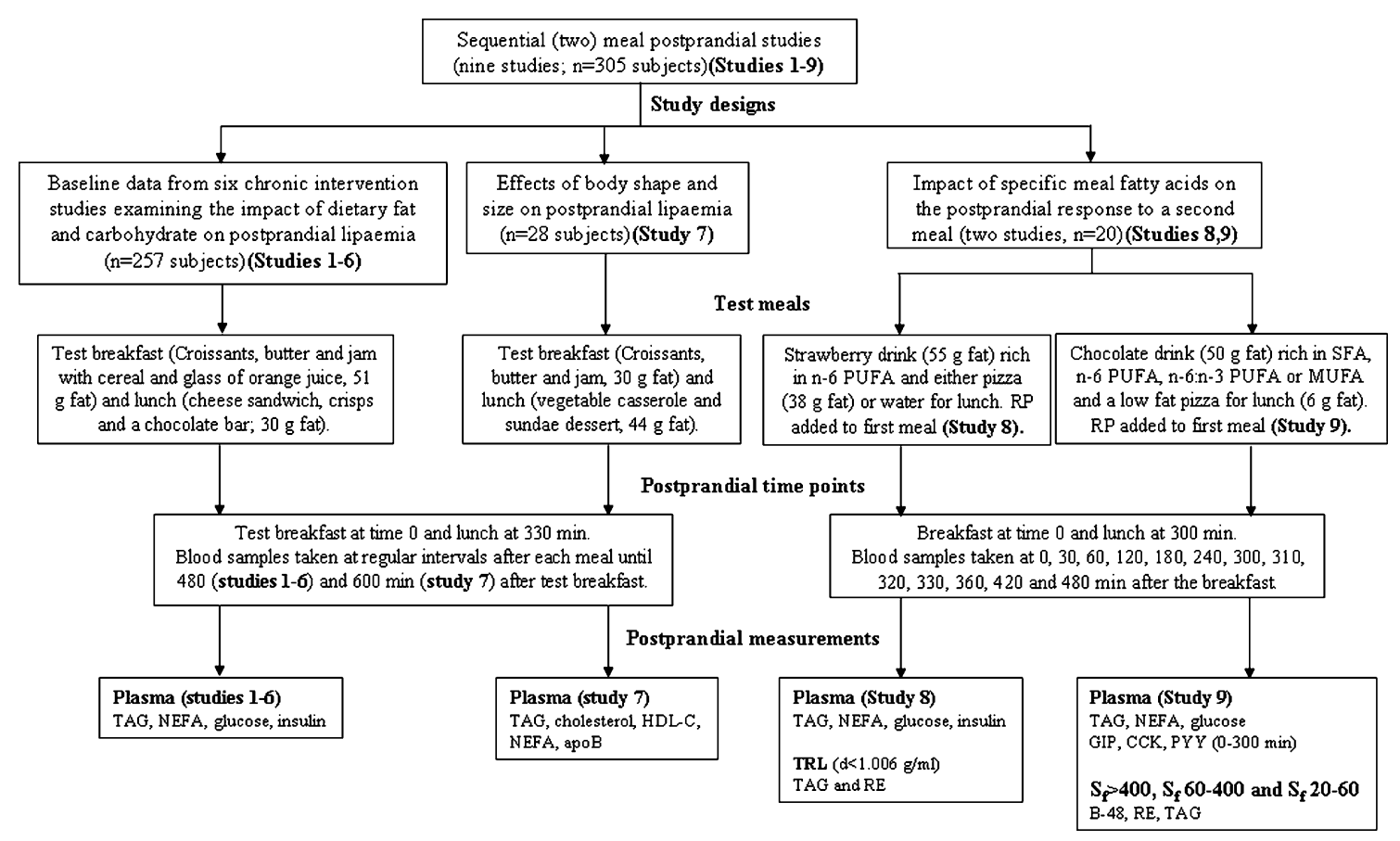

Fig. 1 Overview of the sequential (two) meal postprandial protocols. apoB apolipoprotein B, $C C K$ cholecystokinin, GIP glucose dependent insulinotrophic polypeptide, $H D L-C$ high density lipoprotein cholesterol, MUFA monounsaturated fatty acids, $N E F A$ non-esterified fatty

taken. The standard test breakfast (croissants with butter and jam, cereal with milk and a glass of orange juice) was then given which was consumed within 20 min. Blood samples were collected at regular intervals until $330 \mathrm{~min}$ after the test breakfast when a second meal (a cheese sandwich, packet of crisps and chocolate bar) was given and blood samples collected until $480 \mathrm{~min}$ after the test breakfast. On average 12 blood samples were taken during the postprandial investigation. The details of the test breakfast and lunch meals are shown in Fig. 1. The nutritional composition of the test breakfast was $4.2 \mathrm{MJ}$ energy, $51 \mathrm{~g}$ fat, $125 \mathrm{~g}$ carbohydrate and $19 \mathrm{~g}$ protein and the test lunch was 2.6 MJ energy, $30 \mathrm{~g}$ fat, $79 \mathrm{~g}$ carbohydrate and $15 \mathrm{~g}$ protein. The type of fat contained within the test meals was predominately saturated, with $29 \mathrm{~g}$ of saturated fatty acids (SFA) in the breakfast and $14 \mathrm{~g}$ of SFA in the lunch. No other food or drink except water and decaffeinated sugarfree drinks was allowed during the study day.

Plasma TAG, non-esterified fatty acids (NEFA), glucose and insulin were measured in the fasting and postprandial blood samples.

DNA extraction and genotyping DNA was isolated from the buffy coat layer of $10 \mathrm{ml}$ of EDTA blood using a kit from Qiagen. Allelic discrimination of genes variants of selected apolipoproteins, transfer proteins and lipolytic enzymes involved in the metabolism of TRLs in the acids, $P U F A$ polyunsaturated fatty acids, $P Y Y$ peptide $Y Y, R E$ retinyl ester, $R P$ retinyl palmitate, $S_{\mathrm{f}}$ Svedberg flotation rate, $S F A$ saturated fatty acids, $T A G$ triacylglycerol, $T R L$ triacylglycerol-rich lipoprotein

circulation (Table 2) was conducted using Assay-onDemand SNP genotyping assays (Applied Biosystems). The apoliprotein (apo) A5 variants haplotype (A5*1, A5*2 and $\mathrm{A} 5 * 3$ ) was determined from the alleles for the apoA5 SNPs rs3135506 and rs662799 [13] and the apoE haplotype (E2/E2, E2/E3, E2/E4, E3/E3, E3/E4 and E4/E4) from the alleles for the apoE SNPs rs7412 and rs429358.

Subject and outcome summary A total of $n=257$ individuals underwent the same baseline sequential meal postprandial assessment (studies 1-6), with data for $n=19$ subjects removed from the postprandial dataset as a result of incomplete subject attribute information (age, BMI, gender, blood pressure, fasting lipids or glucose concentration). Therefore, data were available for $n=238$ subjects, of which $n=140$ were males and $n=98$ were females. Detailed postprandial responses are available for plasma TAG $(n=238)$, NEFA $(n=184)$, glucose $(n=158)$ and insulin $(n=61)$ for this sequential meal postprandial dataset.

Single meal studies

Six single meal postprandial studies have examined the effects of meal fatty acids on postprandial lipaemia, in particular their impact on TRL concentration and composition. In general, these studies have examined: 
Table 2 Single nucleotide polymorphisms (SNPs) determined in our sequential meal postprandial dataset

\begin{tabular}{l}
\hline Apoliproteins \\
ApoA-IV \\
ApoA-V \\
ApoB \\
ApoB signal peptide (27/24) \\
ApoC-III \\
ApoE \\
Enzymes and proteins \\
Cholesterol ester transfer protein \\
Intestinal fatty acid binding protein 2 \\
Lipoprotein lipase \\
Microsomal triglyceride transfer protein \\
Hormones, transcription factors and receptors \\
Oestrogen receptor 1 \\
Insulin \\
Insulin receptor substrate 1 \\
Leptin receptor \\
Peroxisome proliferator activated receptor alpha \\
Tumour necrosis factor
\end{tabular}

(a) the acute effects of differing amounts of monounsaturated fatty acids (MUFA) on chylomicron concentrations in young healthy men from the UK $(n=15)$, Ireland $(n=15)$ and Greece $(n=44)$ and middleaged men from the UK $(n=15)[14,15,19,35,41]$ (studies 10 and 11),

(b) the chronic effects of MUFA intake on postprandial lipaemia in young men with a family history of CHD from the UK $(n=13)$ and Ireland $(n=10)$ [36], healthy middle-aged men $(n=30)$ (unpublished) and students residing in fully catered halls of residence ( $n=25$ ) [38] (studies 12-14),

(c) the effects of meals enriched in SFA, polyunsaturated fatty acids (PUFA) and MUFA on the lipid and apolipoprotein composition of TRL fractions in middle-aged men $(n=10)[20,21]$ (study 15$)$.

A simplified overview of the study designs including test meals, blood sampling and postprandial measurements are shown in Fig. 2, with detailed information included within the study publications. The data from these single meal studies will be used to develop integrative mathematical models of postprandial lipoprotein metabolism.

Analyses of the postprandial data

\section{Summary measures of the postprandial data}

The postprandial responses for the metabolites will be expressed as (a) area under the postprandial curve (area under the curve (AUC)) calculated using the trapezoidal rule, (b) incremental AUC (IAUC) calculated as AUC minus fasting concentrations, (c) maximum concentration reached during the postprandial period $(\operatorname{maxC})$ and $(d)$ time to reach maxC (TTM). Due to the nature of the postprandial NEFA curve with an initial drop in NEFA concentrations after the meal, attributable to insulin stimulated inhibition of adipose tissue TAG hydrolysis, NEFA AUC calculated over the $480 \mathrm{~min}$ time period is difficult to interpret. Therefore, the NEFA AUC and IAUC responses will be calculated from the time of suppression (120 min after a fat-rich meal) to the end of the postprandial period. Other summary measures for NEFA will include the percentage NEFA suppression from baseline (an index of insulin sensitivity), minimum concentration reached during the postprandial period $(\operatorname{minC})$ and time to reach minC.

\section{Statistical analyses}

Independent $t$ tests will be used to determine differences in the subject attribute data and postprandial summary measures between the subgroups, with the data for the postprandial time course analysed using general linear models such as a repeated measures analysis of variance (ANOVA).

Statistical associations between the subjects attribute information (age, gender, genotype, anthropometric and biochemical characteristics) and the summary measures of the postprandial metabolites will be performed using simple bivariate correlations. Multivariate regression will be used to determine the independent associations, with partial $R^{2}$ calculated to determine the percentages of variation in the summary measures explained by the independent variables (subject attributes).

\section{Mathematical models of postprandial lipoprotein metabolism}

The creation and utilisation of a database of human postprandial responses to typical fat containing meals as well as the close collaboration between experimentalists and modellers, will enable the development of mathematical and informatics tools to provide a greater understanding of the complex metabolic processes which occur during postprandial lipaemia. Typically, postprandial responses are characterised in terms of summary measures such as AUC and TTM, the sensitivity of which are often determined by the number of blood sampling points during the postprandial assessment. The application of simple compartmental models as well as more sophisticated models will provide a more in-depth understanding of the metabolic determinants (formation, secretion and clearance of TRLs) of the observed variability in the postprandial profile between individuals. 


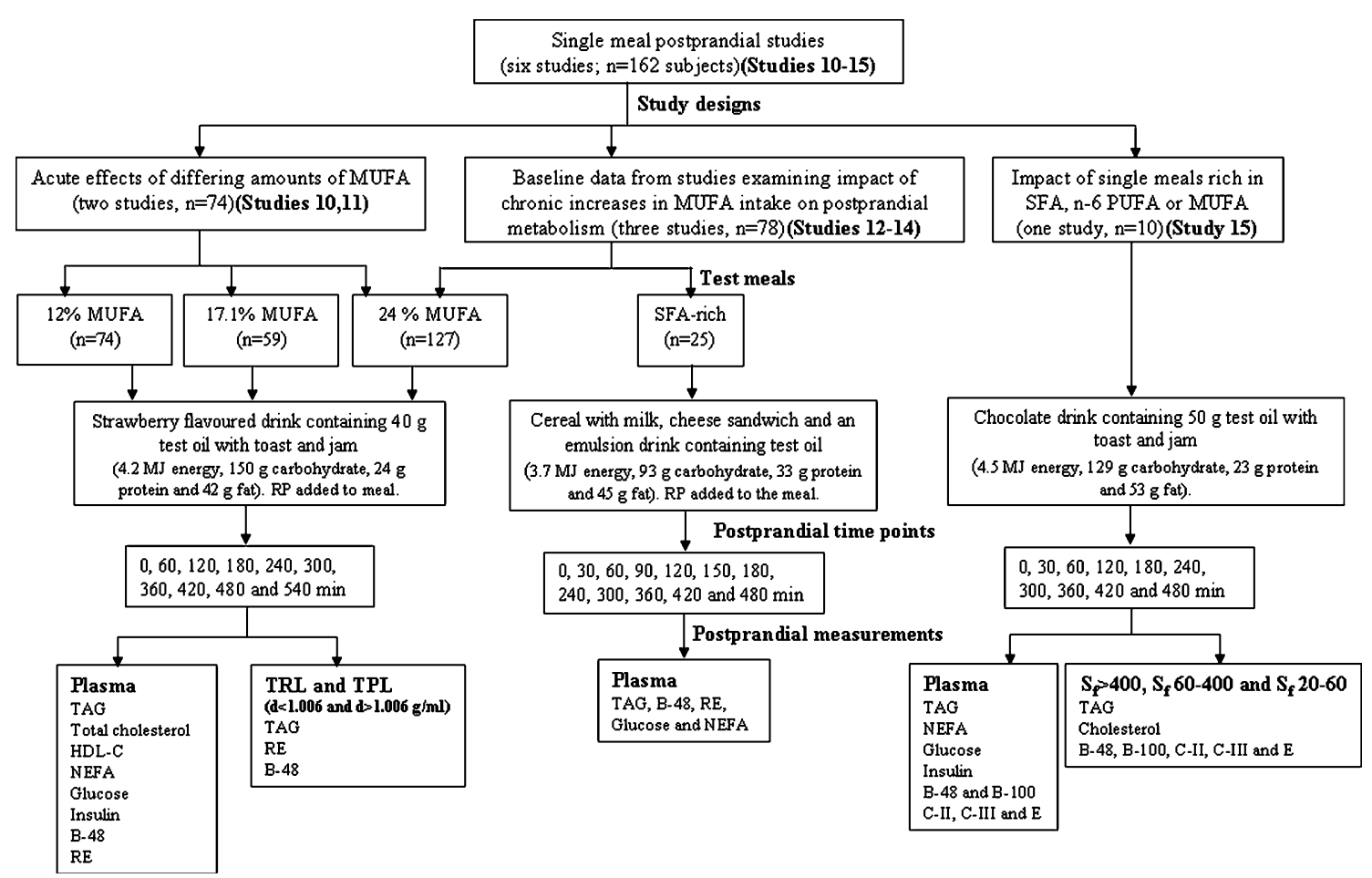

Fig. 2 Overview of the single meal postprandial protocols. apo apolipoprotein, $H D L-C$ high density lipoprotein cholesterol, MUFA monounsaturated fatty acids, NEFA non-esterified fatty acids, $P U F A$ polyunsaturated fatty acids, $R E$ retinyl ester, $R P$ retinyl palmitate,

Advanced data processing methods (e.g., machine learning techniques) are another approach to analysing postprandial responses to typical meals, which is made possible by the large subject numbers in the DISRUPT dataset. The application of such approaches is growing in biology $[30,40]$ as sufficiently large numbers of wellcharacterised subjects are available to produce meaningful and reliable results. Clustering algorithms can also be used to identify subgroups of the population with distinct postprandial responses as well as specific features of the response not obvious from aggregated results. Another common set of techniques are classification algorithms, which can be used to predict the likely 'class' of meal response into which a particular subject will be categorised based on their clinical and biochemical characteristics. These techniques do not rely on a detailed understanding of the physiological response to a meal, but do require sufficiently large subject numbers to produce informative results. Preliminary analyses of the DISRUPT dataset has shown that these techniques have promised in both predicting postprandial responses and revealing distinct responses, which up until now would have been hidden in the average data.

Furthermore, the derived models can be used to design future postprandial investigations (including optimum sampling frequency and time points) test as well as to
$S_{\mathrm{f}}$ Svedberg flotation rate, $S F A$ saturated fatty acids, TAG triacylglycerol, TRL triacylglycerol-poor lipoprotein, TRL triacylglycerolrich lipoprotein

generate new research hypotheses and identify new more informative measures of postprandial responses to single and multiple meals. Undoubtedly, no single dataset will be sufficiently rich to provide a complete description of whole body lipid or glucose metabolism; however, such initiatives and the developed models can provide an excellent starting point for supporting the optimum design and prioritisation of future postprandial studies.

\section{Preliminary findings from the sequential meal postprandial dataset}

Impact of gender on the postprandial TAG response

The mean age, BMI, blood pressure and fasting metabolites for the male and female groups are shown in Table 3. Significant differences in BMI, blood pressure, fasting TAG, HDL-C, LDL-C and glucose were evident between the gender groups $(P<0.001)$.

The magnitude of the postprandial TAG response was significantly different between the gender groups, with a lower AUC (39\%) and IAUC (44\%) in the female group $(P<0.0001$, Table 3$)$. In addition, the TTM was significantly earlier and the maxC significantly lower in the female compared with the male group $(P<0.008)$. 
Table 3 Subject characteristics and postprandial TAG summary measures in the male and female groups and according to age group in the male subjects

\begin{tabular}{|c|c|c|c|c|c|c|}
\hline & Females & Males & $P=($ gender $)$ & Men $<50$ years & Men $\geq 50$ years & $P=($ age $)$ \\
\hline \multicolumn{7}{|l|}{ Subject characteristics } \\
\hline Age & $53.0 \pm 10.8$ & $52.2 \pm 10.8$ & NS & $41.7 \pm 5.4$ & $60.0 \pm 6.3$ & \\
\hline Body mass index $\left(\mathrm{kg} / \mathrm{m}^{2}\right)$ & $25.2 \pm 3.3$ & $26.6 \pm 2.4$ & 0.0002 & $26.3 \pm 2.1$ & $26.9 \pm 2.6$ & NS \\
\hline \multicolumn{7}{|l|}{ Blood Pressure (mmHg) } \\
\hline Systolic & $125 \pm 16$ & $136 \pm 18$ & $<0.0001$ & $130 \pm 15$ & $141 \pm 19$ & 0.0004 \\
\hline Diastolic & $75 \pm 9$ & $84 \pm 11$ & $<0.0001$ & $82 \pm 10$ & $86 \pm 10$ & 0.025 \\
\hline \multicolumn{7}{|l|}{ Biochemical data (mmol/L) } \\
\hline Total cholesterol & $5.65 \pm 1.04$ & $5.92 \pm 1.04$ & NS & $5.69 \pm 0.99$ & $6.09 \pm 1.05$ & 0.026 \\
\hline HDL cholesterol & $1.63 \pm 0.39$ & $1.13 \pm 0.29$ & $<0.0001$ & $1.14 \pm 0.31$ & $1.12 \pm 0.27$ & NS \\
\hline LDL cholesterol & $3.50 \pm 1.04$ & $3.93 \pm 0.96$ & 0.001 & $3.72 \pm 0.90$ & $4.09 \pm 0.98$ & 0.026 \\
\hline Triacylglycerol & $1.17 \pm 0.41$ & $1.91 \pm 0.88$ & $<0.0001$ & $1.92 \pm 0.93$ & $1.91 \pm 0.85$ & NS \\
\hline Glucose & $4.84 \pm 0.46$ & $5.30 \pm 0.53$ & $<0.0001$ & $5.26 \pm 0.53$ & $5.32 \pm 0.53$ & NS \\
\hline \multicolumn{7}{|l|}{ Postprandial TAG data } \\
\hline $\operatorname{AUC}(\mathrm{mmol} / \mathrm{L} \times 480 \mathrm{~min})$ & $830.0 \pm 303.5$ & $1,349.2 \pm 589.5$ & $<0.0001$ & $1,356.5 \pm 648.6$ & $1,343.8 \pm 545.1$ & NS \\
\hline IAUC $(\mathrm{mmol} / \mathrm{L} \times 480 \mathrm{~min})$ & $232.9 \pm 150.0$ & $413.1 \pm 239.8$ & $<0.0001$ & $450.3 \pm 278.9$ & $385.3 \pm 203.1$ & NS \\
\hline $\operatorname{MaxC}(\mathrm{mmol} / \mathrm{L})$ & $2.46 \pm 0.87$ & $3.95 \pm 1.79$ & $<0.0001$ & $4.05 \pm 2.09$ & $3.87 \pm 1.53$ & NS \\
\hline TTM (min) & $366.7 \pm 75.7$ & $392.5 \pm 70.8$ & 0.0076 & $392.3 \pm 71.1$ & $392.7 \pm 71.0$ & NS \\
\hline
\end{tabular}

Values represent mean $\pm \mathrm{SD}$

$A U C$ area under the curve, $H D L$ high density lipoprotein, $I A U C$ incremental area under the curve, $L D L$ low density lipoprotein $M a x C$ maximum TAG concentration, $N S$ not significant, TTM time to reach maxC

\section{Multivariate regression analysis}

Males BMI was shown to be inversely associated with both the TAG AUC $(P=0.008)$ and maxC $(P=0.007)$. Fasting TAG was positively associated with the TAG AUC $(P<0.0001)$, IAUC $(P=0.009)$ and $\operatorname{maxC}(P<0.0001)$, whereas HDL-C was inversely associated with the TAG AUC $\quad(P=0.009), \quad \operatorname{maxC} \quad(P=0.033)$ and TTM $(P=0.006)$. LDL-C was positively associated with TAG AUC $(P=0.019)$ and glucose was positively associated with the TAG AUC $(P=0.0005)$, IAUC $(P=0.005)$ and $\operatorname{maxC}(P=0.002)$.

Females In contrast to the males, BMI was positively associated with the TAG AUC $(P=0.003)$ and maxC $(P=0.014)$. Age was shown to be positively associated with the TAG IAUC $(P=0.019)$ and TTM $(P=0.029)$. Fasting TAG was positively associated with the TAG AUC $(\mathrm{P}<0.0001)$, maxC $(P<0.0001)$ and TTM $(P=0.007)$ and HDL inversely associated with the IAUC $(P=0.033)$.

Impact of age on the postprandial TAG response in males

We have recently reported differences in the postprandial TAG response in our female group sub-divided according to menopausal status [22], with a more exaggerated plasma
TAG response observed after the second meal in the postmenopausal compared with the premenopausal women. Multivariate regression analysis revealed age to be independently associated with the IAUC only in the premenopausal women, accounting for a greater proportion of the variability in the IAUC than the fasting TAG concentration. In order to gain an insight into whether this finding was a gender specific effect, male subjects were divided into two groups, men $<50$ years $(n=50)$ and men $\geq 50$ years $(n=80)$ in the present analysis. The younger men were shown to have significantly lower systolic and diastolic blood pressure, fasting total cholesterol and LDL-C concentrations $(P<0.03)$. There were no differences in the fasting TAG concentration or any of the summary measures of the postprandial TAG response between the male groups (Table 3 ).

\section{Multivariate regression analysis}

Interestingly, BMI was inversely associated with TAG AUC $(P=0.037)$ and $\operatorname{maxC}(P=0.011)$ in men $<50$ years, but not in the older subgroup.

In both male groups, fasting TAG was positively associated with the TAG AUC $(P<0.0001)$ and maxC $(P<0.0005)$. In men $\geq 50$ years, fasting LDL-C was shown to be positively associated with the TAG AUC $(P=0.0004)$, IAUC $(P=0.004)$ and $\operatorname{maxC}(P=0.004)$. 
HDL-C was inversely associated with TTM in the men $<50$ years $(P=0.011)$ and with $\operatorname{AUC}(P=0.017)$ and $\operatorname{maxC}(P=0.026)$ in men $\geq 50$ years. Glucose was positively associated with TAG AUC $(P=0.002)$, IAUC $(P=0.023)$ and $\operatorname{maxC}(P=0.015)$ in men $<50$ years and with the AUC $(P=0.035)$ and $\operatorname{maxC}(P=0.03)$ in men $\geq 50$ years.

\section{Conclusions}

The key features of the DISRUPT postprandial database are (1) large number of both male and female subjects aged between 20 and 75 years which have undertaken the same detailed postprandial investigations, in particular the sequential meal postprandial protocol, (2) comprehensive subject attribute information such as age, gender, menopausal status, blood pressure and fasting biochemical information (lipids and glucose) (3) frequent blood sampling during the postprandial period (at least 10 blood samples taken over an 8-h study day), (4) measurement of a wide range of postprandial outcome measures such as insulin, glucose, NEFA, apolipoproteins (B-48, B-100, C-II, C-III and E) and retinyl ester (marker of intestinally derived lipoproteins), in addition to TAG concentrations, (5) detailed compositional analysis of TRL fractions, (6) availability of genotyping data for a selection of apolipoproteins, enzymes, proteins, hormones, transcription factors and receptors which have been reported to influence postprandial lipaemia [31], and (7) the potential to expand this database to include postprandial data collected by other groups working in this field.

Our preliminary pooled data analysis (presented in "Preliminary findings from the sequential meal postprandial dataset" section) has revealed both confirmatory and novel observations with respect to the impact of gender and age on the postprandial TAG response. In agreement with the literature, we have found a greater postprandial TAG response in males compared with females [7, 12], with regression analyses indicating inverse associations between fasting TAG (positive) and HDL-C (negative) with the postprandial TAG response [1]. Novel findings include the differences in the directions of the relationships between various subject characteristics of the male and females and the summary measures of the postprandial TAG response. In particular, an inverse relationship between BMI and the TAG AUC was observed in the males suggesting a more favourable TAG clearance after the sequential test meals in those with a greater fat mass. Interestingly, when the male group was sub-divided into men $<50$ years and men $\geq 50$ years, this association was only observed in the men $<50$ years even though BMI was similar in these two groups of men. A possible explanation for this relationship between BMI and the postprandial TAG response, which differs from many reports in the literature [8, 9, 37], is that for younger men with normal insulin sensitivity, the capacity for TAG clearance is partly determined by the area of the adipose tissue vascular bed which will be greater in individuals with a higher BMI. With ageing and increasing insensitivity to insulin, which will be greater in those with a higher fat mass, this relationship disappears. However, as reported previously, it should be noted that subdivision of the female participants according to menopausal status revealed BMI to be positively associated with TAG AUC in the premenopausal women only [22] and it is unclear at this stage why the age and BMI dependent relationships should differ according to gender. Further analyses will include determination of the most important subject attributes that influence the magnitude and duration of the postprandial lipid and glucose responses and the identification of qualitative differences in the profile of the postprandial responses between subgroups of the population, using machine learning methods. In addition to significantly contributing to our current understanding of the determinants of the postprandial metabolism, output from the pooled datasets will help to identify gaps in our current knowledge and help to formulate future postprandial investigations.

Acknowledgments The authors are grateful to Nicky Wilkinson for advice on data protection issues and Adrian Smith and Dr. Cesar Pichardo-Almarza for testing the on-line analysis tools and reporting tools during the development of the DISRUPT postprandial database. We also thank Drs. Pieter Groot, Charlotte Walden and Cesar Pichardo-Almarza for proof-reading of the manuscript. The DISRUPT project was funded by a BBSRC Industry Interchange Program (IIP/ 0307/009).

Conflict of interest statement The authors had no conflicts of interest.

\section{References}

1. Austin MA, Hokanson JE, Edwards KL (1998) Hypertriglyceridamia as a cardiovascular risk factor. Am J Cardiol 81:7B-12B

2. Bansal S, Buring JE, Rifai N, Sacks FM, Ridker PM (2007) Fasting compared with nonfasting triglycerides and risk of cardiovascular events in women. JAMA 298:309-316

3. Brady LM, Lovegrove SS, Lesauvage SVM, Gower BA, Minihane AM, Williams CM, Lovegrove JA (2004) Increased n-6 polyunsaturated fatty acids do not attenuate the effects of longchain n-3 polyunsaturated fatty acids on insulin sensitivity or triacylglycerol reduction in Indian Asians. Am J Clin Nutr 79:983-991

4. Buttriss JL (2006) Hanging on the coat tails of the obesity epidemic: will metabolic syndrome be the next public health crisis and does nutrition have a role in prevention? J Sci Food Agric $86: 2285-2289$

5. Codd EF (1970) A relational model of data for large shared data banks. Commun ACM 13:377-387 
6. Codd EF (1971) Further normalization of the data base relational model. Courant Computer Science Symposia Series 6, Data Base Systems, New York City

7. Cohn JS, McNamra JR, Cohn SD, Ordovas JM, Schaefer EJ (1988) Postprandial plasma lipoprotein changes in human subjects of different ages. J Lipid Res 29:469-479

8. Couillard C, Bergeron N, Prud'homme D, Bergeron J, Tremblay A, Bouchard C, Mauriège P, Després JP (1998) Postprandial triglyceride response in visceral obesity in men. Diabetes 47:953960

9. Després JP, Moorjani S, Lupien PJ, Tremblay A, Nadeau A, Bouchard C (1990) Regional distribution of body fat, plasma lipoproteins, and cardiovascular disease. Arteriosclerosis 10:497511

10. Finnegan YE, Minihane AM, Leigh-Firbank EC, Kew S, Meijer GW, Muggli R, Calder PC, Williams CM (2003) Plant- and marine derived $n-3$ polyunsaturated fatty acids have differential effects on fasting and postprandial blood lipid concentrations and on the susceptibility of LDL to oxidative modification in moderately hyperlipidaemic subjects. Am J Clin Nutr 77:783-795

11. Friedewald WT, Levy RI, Fredrickson DS (1972) Estimation of the concentration of LDL cholesterol in plasma, without the use of the preparative ultracentrifuge. Clin Chem 18:499-502

12. Halkes CJM, Castro Cabezas M, van Wijk JPH, Erkelens DW (2001) Gender differences in diurnal hypertriglyceridemia in lean and overweight subjects. Int J Obes 25:1767-1774

13. Henneman P, Schaap FG, Havekes LM, Rensen PCN, Frants RR, van Tol A, Hatori H, Smelt AHM, van Dijk KW (2007) Plasma apoAV levels are markedly elevated in severe hypertriglyceridemia and positively correlated with the apoA5 S19 W polymorphism. Atherosclerosis 193:129-134

14. Jackson KG, Zampelas A, Knapper JME, Culverwell CC, Wright J, Gould BJ, Williams CM (1999) Lack of influence of meal fatty acid composition on the contribution of intestinally derived lipoproteins to postprandial lipaemia. Brit J Nutr 81:51-57

15. Jackson KG, Zampelas A, Knapper JME, Roche HM, Gibney MJ, Kafatos A, Gould BJ, Wright JW, Williams CM (2000) Differences in glucose dependent insulinotrophic polypeptide hormone and hepatic lipase in subjects of southern and northern Europe: implications for postprandial lipemia? Am J Clin Nutr 71:13-20

16. Jackson KG, Robertson MD, Fielding BA, Frayn KN, Williams CM (2001) Second meal effect: modified sham feeding does not provoke the release of stored triacylglycerol from a previous high-fat meal. Brit J Nutr 85:149-156

17. Jackson KG, Robertson MD, Fielding BA, Frayn KN, Williams CM (2002) Olive oil increases the number of triacylglycerol-rich chylomicron particles compared with other oils: an effect retained when a second standard meal is fed. Am J Clin Nutr 76:942-949

18. Jackson KG, Robertson MD, Fielding BA, Frayn KN, Williams CM (2002) Measurement of apolipoprotein B-48 in the Svedberg flotation rate $\left(S_{\mathrm{f}}\right)>400, \mathrm{~S}_{\mathrm{f}} 60-400$ and $\mathrm{S}_{\mathrm{f}}$ 20-60 lipoprotein fractions reveal novel findings with respect to the effects of dietary fatty acids on triacylglycerol-rich lipoproteins in postmenopausal women. Clin Sci (Lond) 103:227-237

19. Jackson KG, Knapper-Francis JME, Morgan LM, Webb DH, Zampelas A, Williams CM (2003) Exaggerated postprandial lipaemia and lower postheparin lipoprotein lipase activity in middle-aged men. Clin Sci 105:457-466

20. Jackson KG, Wolstencroft EJ, Bateman PA, Yaqoob P, Williams CM (2005) Greater enrichment of triacylglycerol-rich lipoproteins with apolipoproteins E and C-III after meals rich in saturated fatty acids than after meals rich in unsaturated fatty acids. Am J Clin Nutr 81:25-34

21. Jackson KG, Wolstencroft EJ, Bateman PA, Yaqoob P, Williams CM (2005) Acute effects of meal fatty acids on postprandial NEFA, glucose and apolipoprotein E response: implications for insulin sensitivity and lipoprotein regulation? Brit J Nutr 93:693700

22. Jackson KG, Abraham EC, Smith AM, Murray P, O’Malley B, Williams CM, Minihane AM (2009) Impact of age and menopausal status on the postprandial triacylglycerol response in healthy women. Atherosclerosis. doi:10.1016/j.atherosclerosis.2009.06.030

23. Lairon D, Lopez-Miranda J, Williams C (2007) Methodology for studying postprandial lipid metabolism. Eur J Clin Nutr 61:11451161

24. Leigh-Firbank EC, Minihane AM, Leake DS, Wright JW, Murphy MC, Griffin BA, Williams CM (2002) Eicosapentaenoic acid and docosahexaenoic acid from fish oils: differential associations with lipid responses. Brit J Nutr 87:435-445

25. Lopez-Miranda J, Williams C, Lairon D (2007) Dietary, physiological, genetic and pathological influences on postprandial lipid metabolism. Brit J Nutr 98:458-473

26. Lovegrove JA, Chlohessy A, Milon H, Williams CM (2000) Modest doses of $\beta$-glucan do not reduce concentrations of potentially atherogenic lipoproteins. Am J Clin Nutr 72:49-55

27. Lovegrove JA, Silva KDRR, Wright JW, Williams CM (2002) Adiposity, insulin and lipid metabolism in postmenopausal women. Int J Obes 26:475-486

28. Minihane AM, Khan S, Leigh-Firbank EC, Talmud P, Wright JW, Murphy MC, Griffin BA, Williams CM (2000) ApoE polymorphism and fish oil supplementation in subjects with an atherogenic lipoprotein phenotype. Arterioscler Thromb Vasc Biol 20:1990-1997

29. Nordestgaard BG, Benn M, Schnohr P, Tybjærg-Hansen A (2007) Nonfasting triglycerides and risk of myocardial infaction, ischamic heart disease, and death in men and women. JAMA 298:299-308

30. Pencina MJ, Millen BE, Hayes LJ, D'Agostino RB (2008) Performance of a method for identifying the unique dietary patterns of adult women and men: the Framingham nutrition studies. J Am Diet Assoc 108:1453-1460

31. Perez-Martinez P, Lopez-Miranda J, Perez-Jimenez F, Ordovas JM (2008) Influence of genetic factors in the modulation of postprandial lipemia. Atheroscler Suppl 9:49-55

32. Robertson MD, Jackson KG, Fielding BA, Williams CM, Frayn KN (2001) Prolonged effects of modified sham feeding on energy substrate mobilization. Am J Clin Nutr 73:111-117

33. Robertson MD, Jackson KG, Fielding BA, Morgan LM, Williams CM, Frayn KN (2002) Acute effects of meal fatty acid composition on insulin sensitivity in healthy post-menopausal women. Brit J Nutr 88:635-640

34. Robertson MD, Jackson KG, Fielding BA, Morgan LM, Williams CM, Frayn KN (2002) Acute ingestion of triacylglycerol rich in n-3 polyunsaturated fatty acids results in rapid gastric emptying. Am J Clin Nutr 76:232-237

35. Roche HM, Zampelas A, Jackson KG, Williams CM, Gibney MJ (1998) The effect of test meal monounsaturated fatty acid: saturated fatty acid ratio on postprandial lipid metabolism. Brit J Nutr 79:419-424

36. Roche HM, Zampelas A, Knapper JME, Webb D, Brooks C, Jackson KG, Wright JW, Gould BJ, Kafatos A, Gibney MJ, Williams CM (1998) Effect of the long-term olive oil dietary intervention on postprandial triacylglycerol and factor VII metabolism. Am J Clin Nutr 68:552-560

37. Sattar N, Tan CE, Han TS, Forster L, Lean MEJ, Shepherd J, Packard CJ (1998) Associations of indices of adiposity with atherogenic lipoprotein subfractions. Int $\mathbf{J}$ Obes Relat Metab Disord 22:432-439

38. Silva KDRR, Kelly CNM, Jones AE, Smith RD, Wootton SA, Miller GJ, Williams CM (2003) Chylomicron particle size and number, factor VII activation and dietary monounsaturated fatty acids. Atherosclerosis 166:73-84 
39. Silva KDRR, Wright JW, Williams CM, Lovegrove JA (2005) Meal ingestion provokes entry of lipoproteins containing fat from the previous meal: possible metabolic implications. Eur J Clin Nutr 44:377-383

40. Szczepaniak PS, Lisboa PJG, Kacprzyk J (2000) Fuzzy Systems in Medicine. Berlin. Physica-Verlag. 698 p

41. Zampelas A, Roche H, Kapsokefalou M, Knapper JME, Jackson KG, Pentaris E, Tornaritis M, Gibney MJ, Kafatos A, Gould BJ,
Wright JW, Williams CM (1998) Differences in postprandial lipaemic responses between northern and southern Europeans. Atherosclerosis 139:83-93

42. Zilversmit DB (1979) Atherogenesis-postprandial phenomenon. Circulation 60:473-485 